\title{
Plasma eyepieces for petawatt class lasers
}

Cite as: Phys. Plasmas 27, 023109 (2020); https://doi.org/10.1063/1.5116416

Submitted: 25 June 2019 . Accepted: 30 January 2020 . Published Online: 13 February 2020

(Di) Ming Zeng, (i) Alberto Martinez de la Ossa, (D) Kristjan Poder, and Jens Osterhoff
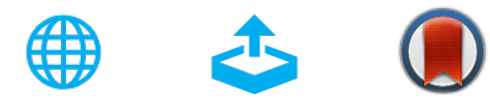

View Online

Export Citation

CrossMark

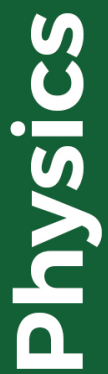

\section{ARTICLES YOU MAY BE INTERESTED IN}

Perspectives on the generation of electron beams from plasma-based accelerators and their near and long term applications

Physics of Plasmas 27, 070602 (2020); https://doi.org/10.1063/5.0004039

Net energy gain in direct laser acceleration due to enhanced dephasing induced by an applied magnetic field

Physics of Plasmas 27, 023110 (2020); https://doi.org/10.1063/1.5122893

Current status and highlights of the ELI-NP research program

Matter and Radiation at Extremes 5, 024402 (2020); https://doi.org/10.1063/1.5093535

\section{AIP Advances Fluids and Plasmas Collection}




\title{
Plasma eyepieces for petawatt class lasers
}

\author{
Cite as: Phys. Plasmas 27, 023109 (2020); doi: 10.1063/1.5116416 \\ Submitted: 25 June 2019 - Accepted: 30 January 2020 • \\ Published Online: 13 February 2020
}

Ming Zeng, ${ }^{\text {a) }}$ (D) Alberto Martinez de la Ossa, (D Kristjan Poder, (D and Jens Osterhoff

\author{
AFFILIATIONS \\ Deutsches Elektronen-Synchrotron DESY, 22607 Hamburg, Germany

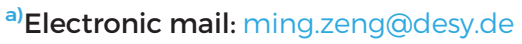

\begin{abstract}
Focusing petawatt class laser beams to a variety of spot sizes for different applications is expensive in cost, labor, and space. In this paper, we propose a plasma lens to flexibly resize the laser beam by utilizing the self-focusing effect of laser in plasmas. Using a fixed conventional focusing system to focus the laser a short distance in front of the plasma, we can adjust the effective laser beam waist within a certain range, with the plasma lens acting as an adjustable eyepiece in a telescope. Such a setup is a powerful tool for laser wakefield accelerator experiments in state-of-the-art petawatt laser projects and allows for scanning focal spot parameters.
\end{abstract}

Published under license by AIP Publishing. https://doi.org/10.1063/1.5116416

\section{INTRODUCTION}

Owing to the remarkable developments of laser technology in the last decades, petawatt-class laser projects are springing up around the world. ${ }^{1}$ For example, the extreme light infrastructure (ELI) project is building several 10 PW laser facilities in Europe, ${ }^{2,3}$ the Vulcan 2020 project is building a $20 \mathrm{PW}$ laser facility in $\mathrm{UK}^{4}$, and the Gwangju Institute of Science and Technology (GIST) in South Korea has reported its establishment of a 4.2 PW laser facility, which has recently opened for user applications. ${ }^{5}$ The Shanghai Institute of Optics and Fine Mechanics (SIOM) has built a 10-PW-level laser system named Shanghai Superintense Ultrafast Laser Facility (SULF-10 PW laser) and is ambitiously aiming for a $100 \mathrm{PW}$ level laser facility named the station of extreme light (SEL).

Manipulation of such powerful lasers is a big challenge due to the lack of high damage-threshold optical materials. The current solution is to use large beam apertures so that the laser power is spread across a large area of the optical element to prevent damage. For example, $\mathrm{SiO}_{2}$ has a damage threshold on the order of $1 \mathrm{~J} / \mathrm{cm}^{2}$ if it is irradiated by a femtosecond laser. ${ }^{8}$ If we assume that the laser pulse duration is $50 \mathrm{fs}$, the wavelength is $800 \mathrm{~nm}$, and the laser beam is spatially perfectly Gaussian, to focus a $1 \mathrm{PW}$ laser beam, a mirror with at least $0.34 \mathrm{~m}$ diameter is required to prevent mirror damage, resulting in high costs for high-quality focusing optics. The cost issue is compounded by the fact that for each laser system, multiple focusing systems are required for different applications: short focal length optics for laser-solid interactions and long focal length ones for laser-gas interactions. A particularly demanding application is laser wakefield acceleration (LWFA), where the laser beam should be focused to a matched spot size that stabilizes laser propagation in the plasma, with the size of this matched spot changing with laser power, plasma density, and plasma channel depth in the external guiding case. ${ }^{9-14}$ For photon-nuclear interaction applications, a changeable laser spot size is also advantageous for maximizing the electron flux. ${ }^{15}$

Besides the requirement of variable laser spot sizes, the long focal length is also challenging. In order to avoid damaging laser optics, the beam near field diameter $D$ must scale as $D \propto \sqrt{P}$ with laser power $P$. The f-number $N \equiv f / D \propto k w_{0}$, where $f$ is the focal length, $w_{0}$ is the laser beam waist at focus, and $k=2 \pi / \lambda$ is the wavenumber for a laser with wavelength $\lambda$. Thus, $f \propto w_{0} \sqrt{P} \propto P / a_{0}$, where $a_{0} \approx 8.5 \times 10^{-10} \lambda(\mu \mathrm{m}) \sqrt{I_{0}\left(\mathrm{~W} / \mathrm{cm}^{2}\right)} \propto \sqrt{P} / w_{0}$ is the normalized laser amplitude and $I_{0}$ is the peak intensity. For laser-solid interactions, radiation-reaction or quantum electrodynamics studies the largest $a_{0}$; thus, a short focal length is required. ${ }^{16-19}$ For LWFA studies, a moderate $a_{0}$ is required even with increasing $P$ to maximize the charge of the accelerated electron beam and the energy gain, ${ }^{12}$ resulting in the focal length $f \propto P$ being extremely long. For example, focal lengths on the order of $10 \mathrm{~m}$ are required for $1 \mathrm{PW}$ lasers, ${ }^{20}$ while for $10 \mathrm{PW}$ (100 PW) lasers, the focal lengths would be on the order of $100 \mathrm{~m}(1 \mathrm{~km})$. With such scales presenting obvious difficulties, a focusing system with a variable focal spot size and small footprint is urgently required.

In this work, we introduce such a telescope system employing the laser self-focusing effect in plasmas. ${ }^{21}$ The scheme is illustrated in Fig. 1. A high power laser beam is prefocused to $z_{0}$ by a conventional focusing system with a focal length of $f_{0}$ to a spot size of $w_{0}$. After propagating a distance $d$, the laser enters the plasma region starting at 


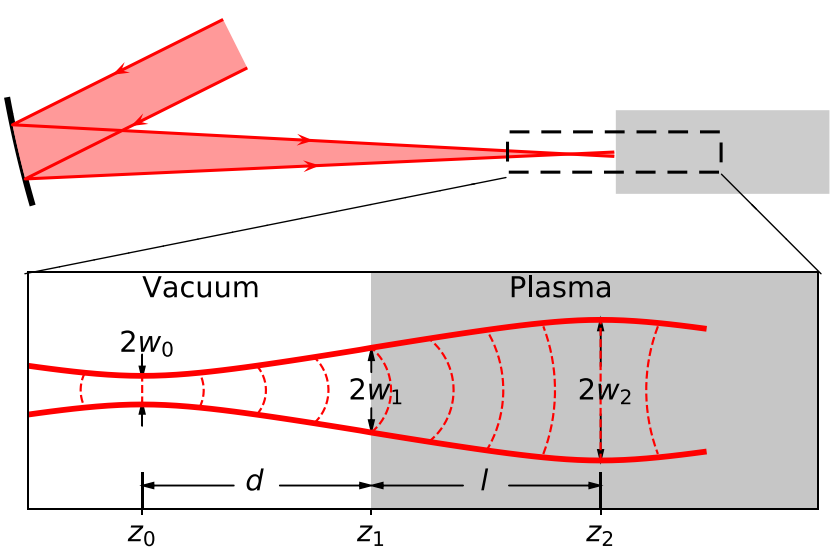

FIG. 1. Illustration of the telescope system. The laser is focused in vacuum to $z=z_{0}$ with a waist of $w_{0}$ by a conventional optical system and then enters a plasma at $z$ $=z_{1}$. The plasma can thereafter reshape the wavefront so that the wavefront becomes flat again at $z=z_{2}$ with the laser size $w=w_{2}$. In the bottom subplot, the solid red curves illustrate the transverse envelope, and the dashed red curves illustrate the wavefront of the laser beam.

$z_{1}$. After another distance $l$, the laser beam is refocused to a spot size $w_{2}$ at $z_{2}$ because of self-focusing effects. ${ }^{21}$ Consequently, the total function of this system is to focus the laser to a spot size of $w_{2}$ within a distance of $L=f_{0}+d+l$, and the plasma acts as the eyepiece of this telescope system. Due to the strong plasma response, $L$ can be much shorter than a conventional focusing system resulting in the same spot size. In addition, $w_{2}$ is adjustable by changing $d, l$, and the plasma density $n_{p}$. Compared to previous studies on plasma lenses for lasers, ${ }^{22-25}$ the herein proposed concept does not require a preformed structure and is preferably suitable for petawatt class lasers.

This paper is organized as follows. In Sec. II, an analytical model describing the plasma eyepiece in the weakly relativistic regime is developed. In Sec. III, an empirical model is found based on particlein-cell (PIC) simulations. The empirical and analytical models are compared in Sec. IV and a full scale LWFA simulation employing the proposed scheme is shown in Sec. V. The adjustment limits and the perspectives for 10 to $100 \mathrm{PW}$ LWFAs are discussed in Sec. VI, and Sec. VII concludes the paper.

\section{WEAKLY RELATIVISTIC SELF-REFOCUSING MODEL}

The evolution of the laser pulse in an underdense plasma in the long pulse and slow profile variation assumptions is given by ${ }^{26}$

$$
\left(\nabla_{\perp}^{2}-i 2 k \partial_{z}\right) \tilde{a}=n\left(\frac{1}{\gamma}-1\right) \tilde{a},
$$

where $z$ is the laser propagation distance, $n$ is the local plasma electron density, and $\gamma$ is the plasma electron Lorentz factor. Normalized units are adopted with densities being normalized to the background plasma density $n_{p}$, wavenumbers to the plasma wavenumber $k_{p}=\sqrt{4 \pi r_{e} n_{p}}$, where $r_{e} \approx 2.82 \times 10^{-15} \mathrm{~m}$ is the classical electron radius, and lengths normalized to the plasma skin depth $k_{p}^{-1}$. Under the paraxial approximation, the cylindrically symmetrical normalized laser vector potential $\tilde{a}$, including the transverse phase modulation but excluding the laser quiver factor $\exp (-i k z+i \omega t)$, is given by

$$
\tilde{a}=a \exp \left(i u r^{2}\right) \exp \left(-\frac{r^{2}}{w^{2}}\right),
$$

where $r$ is radius, $a=a(z)$ is the axial normalized laser vector potential amplitude, $u=u(z)$ is the spatial phase modulation factor (effectively, the radius of the wavefront curvature is $k / 2 u)$, and $w=w(z)$ is the laser spot size.

In general, solving Eq. (1) analytically is difficult. However, it has been found that under weakly relativistic assumptions where the plasma density is unperturbed $n=1$, the approximate solution of Eq. (1) for a linearly polarized laser beam can be obtained by calculus of variations ${ }^{27-29}$ as follows (derivation is given in Appendix A):

$$
\begin{gathered}
|a|^{2} w^{2}=\left|a_{0}\right|^{2} w_{0}^{2}, \\
\frac{d^{2} w}{d z^{2}}=\frac{4}{k^{2} w^{3}}\left(1-\frac{\left|a_{0}\right|^{2} w_{0}^{2}}{32}\right),
\end{gathered}
$$

where Eq. (3) represents the conservation law in the case of negligible energy loss and frequency shift (which is true if the propagation distance is short compared to the pump depletion length) and Eq. (4) describes the evolution of the laser spot size in the weakly relativistic regime $a \leqq 1$. $^{30}$

We apply Eq. (4) to our case, with the initial conditions at the vacuum-plasma interface $\left(z=z_{1}\right)$ reading

$$
\begin{aligned}
\left.w_{1} \equiv w\right|_{z_{1}} & =w_{0} \sqrt{1+\frac{d^{2}}{z_{R}^{2}}} \\
\left.\frac{d w}{d z}\right|_{z_{1}} & =\frac{w_{0}^{2} d}{z_{R}^{2} w_{1}}
\end{aligned}
$$

where $z_{R}=k w_{0}^{2} / 2$ is the Rayleigh length. After integrating Eq. (4), the refocused spot size can be found by requiring $\mathrm{d} w / \mathrm{d} z=0$ and is given by

$$
\left.w_{2} \equiv w\right|_{z_{2}}=w_{0} \sqrt{1+\frac{d^{2}}{z_{R}^{2}} \cdot \frac{1}{1-\left(1+\frac{d^{2}}{z_{R}^{2}}\right) \frac{32}{a_{0}^{2} w_{0}^{2}}}} .
$$

The length of the plasma eyepiece $l$ is given by

$$
l \equiv z_{2}-z_{1}=\frac{d}{\frac{a_{0}^{2} w_{0}^{2}}{32}\left(1+\frac{d^{2}}{z_{R}^{2}}\right)^{-1}-1}
$$

with the limit

$$
d<z_{R} \sqrt{\frac{a_{0}^{2} w_{0}^{2}}{32}-1} \equiv d_{\mathrm{M}} .
$$

Thus, $d_{\mathrm{M}}$ is the upper limit of the prefocusing distance $d$ for selfrefocusing to occur.

Equations (7)-(9) show that in the analytical model the effective laser spot size $w_{2}$ and the plasma eyepiece thickness $l$ are functions of $k, w_{0}, a_{0}$, and $d$, indicating the parameter space to be scanned in the simulation studies in Sec. III.

\section{SIMULATIONS AND EMPIRICAL FORMULAS}

The analytic model presented in Sec. II is valid for $a_{0} \lesssim 1$, while commonly LWFAs operate in the nonlinear regime $\left(a_{0}>1\right)$, where 
plasma electrons are strongly expelled from the laser propagation path, generating the so-called plasma blowout. To examine the behavior of the plasma eyepiece in the blowout regime, three-dimensional (3D) particle-in-cell (PIC) simulations using the code OSIRIS ${ }^{31}$ were performed. To characterize the plasma eyepiece, the refocused spot size $w_{2}$ and plasma eyepiece thickness $l$ were found by scanning a four-dimensional parameter space of $\left(k, w_{0}, a_{0 \text { peak }}, d\right)$, where $a_{0 \text { peak }}$ is the peak normalized laser amplitude along the laser comoving coordinate $\xi=z-c t$, and $\bar{a}_{0}=a_{0 \text { peak }} / 2$ is the averaged value along the laser temporal profile (to compare the analytical model which uses an infinite long pulse with the simulations which use finite laser pulses, we define $\bar{a}_{0}$ as the averaged laser amplitude in the simulations and take $a_{0}=\bar{a}_{0} \equiv a_{0 \text { peak }} / 2$ in all the following discussions). The longitudinal profile of the laser pulse is a bell shape (both the rise and fall envelopes of the laser take the form $10 X^{3}-15 X^{4}+6 X^{5}$ in the range $0 \leq X \leq 1$ where $X=\left|\xi-\xi_{0}\right| / \tau$ and $\xi_{0}$ is the pulse center). The initial full-width-at-half-maximum (FWHM) pulse duration is $\tau_{\mathrm{FWHM}}$ $=4$ (time is normalized to $\left.\omega_{p}^{-1}=k_{p}^{-1} c^{-1}\right)$. The simulations were performed using a window of length 10 , comoving with the laser pulse in the positive $z$ direction with the speed of light. The transverse extent of the simulation box was adjusted to be between $10 w_{2}$ and $12 w_{2}$. After convergence test, the number of cells adopted is shown in Table I. Simulation time steps were set close to the Courant condition to minimize numerical dispersion. The number of macroparticles per cell is 8 , and they are initiated with a thermal momentum of $p_{\mathrm{th}} / m_{e} c=0.01$.

For each simulation, snapshots of the absolute value of the laser electric field are projected to the $x-y$ plane (front view). Twodimensional Gaussian fits are then performed to obtain the spot size $w$ for each snapshot. Example simulation results are shown in Fig. 2. A laser with $k=10$ and $a_{0 \text { peak }}=10$ is focused to $w_{0}=4$ at $z_{0}=-100$. It then enters the plasma region starting at $z_{1}=0$ and self-refocusing occurs. As seen in Fig. 2(a), $w$ reaches a local maximum $w_{2}=8.7$ at $z_{2}=104 \pm 4$ for the half-infinite plasma case, plotted as black squares. In the case of a limited plasma region ending at $z_{2}$ [which is plotted as red circles in Fig. 2(a)] in the region $z>z_{2}$, the laser spot size approximately evolves as

$$
w=w_{0 e} \sqrt{1+\frac{\left(z-z_{0 e}\right)^{2}}{z_{R e}^{2}}},
$$

where $w_{0 e}$ is the effective focal size, $z_{0 e}$ is the effective focal position, and $z_{R e}=k w_{0 e}^{2} / 2$ is the effective Rayleigh length with $k=10$ in this case. The evolution of the spot size in the vacuum of the rear side can be fitted by Eq. (10), resulting in $z_{0 e}=53.8$ and $w_{0 e}=8.0$. The data in the region $z<200$ are ignored in the fit because the far-distance measurement can better represent the beam size due to the

TABLE I. Resolution (longitudinal $\times$ transverse) used for simulations presented in the current work, verified by convergence test.

\begin{tabular}{cc}
\hline \hline$k$ & Number of cells \\
\hline 10 & $512 \times 512^{2}$ \\
20 & $1024 \times 512^{2}$ \\
30 & $1024 \times 512^{2}$ \\
40 & $2048 \times 512^{2}$ \\
\hline
\end{tabular}
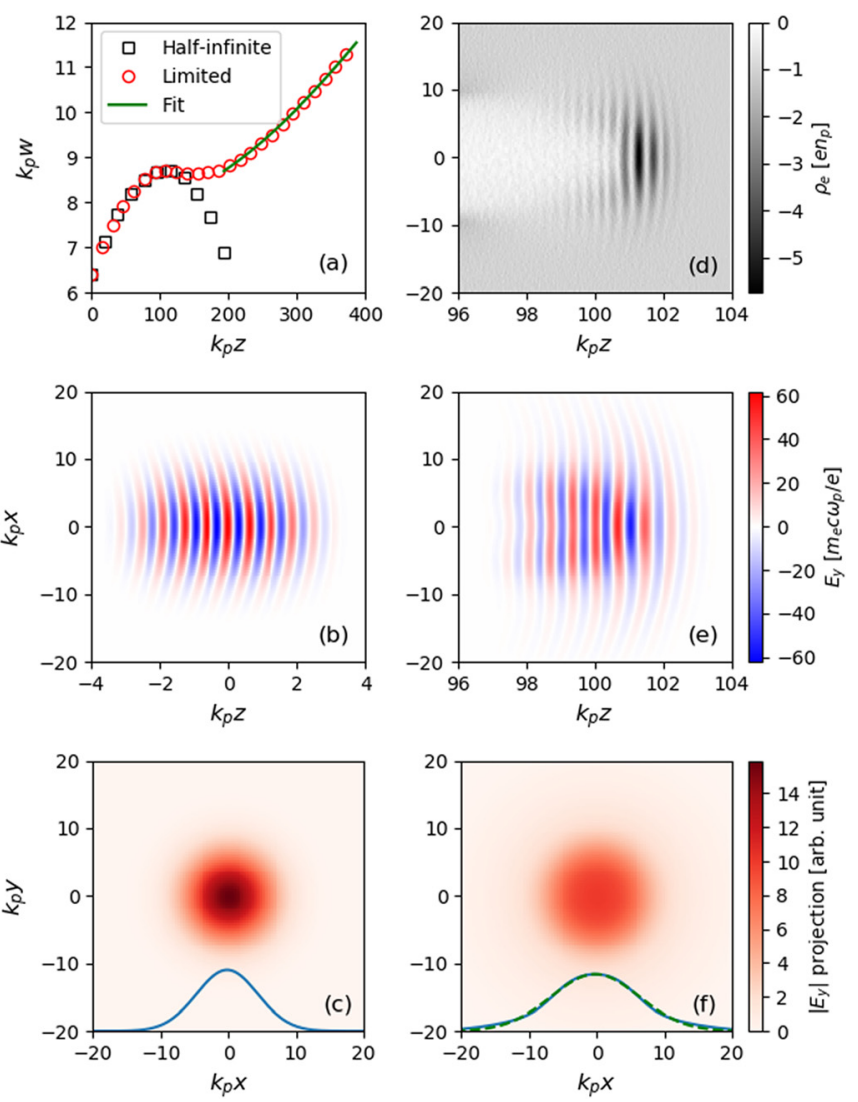

FIG. 2. Example PIC simulations with $k=10, w_{0}=4, a_{0 \text { peak }}=10, d=100$ and plasma region at $z>0$ (thus the laser is prefocused at $z=-100$ ). (a) Spot size $w$ as a function of propagation distance $z$, for a half-infinite plasma which has a uniform profile from $z=0$ to $+\infty$ (black squares) and a limited plasma region from $z=0$ to $z=z_{2}$ (red circles), with $z_{2}=104 \pm 4$ in this case. The green curve is a fit by Eq. (10) for the red circles for $z>200$. (b) Side view (slice at $y=0$ ) of $E_{y}$ at the beginning of the simulation. (c) Front view (projection to the $x-y$ plane) of $\left|E_{y}\right|$ at the beginning of the simulation. (d) Side view of the plasma electron charge density at $z \approx z_{2}$. (e) Side view of $E_{y}$ at $z \approx z_{2}$. (f) Front view of $\left|E_{y}\right|$ at $z \approx z_{2}$. The solid blue lines in (c) and $(f)$ are the line projections of the front views, and the dashed green line in (f) is the Gaussian fit of the blue solid line with the coefficient of determination $R^{2}=0.996$, showing that the laser envelop only slightly deviates from a Gaussian profile.

nonperfectly Gaussian profile of the laser beam. The mismatch $z_{0 e} \neq$ $z_{2}$ and $w_{0 e} \neq w_{2}$ is due to the laser transverse profile not being perfectly Gaussian at $z=z_{2}$ (containing higher-order modes) as highlighted in Figs. 2(e) and 2(f). However, with only a 10\% difference between the spot sizes, $w_{2}$ is used as the effective spot size in the following.

\section{A. Effective focal size vs prefocusing distance}

The dependence of the ratio $w_{2} / w_{0}$ on the prefocusing distance $d$ for varying laser peak amplitudes at focus $a_{0 \text { peak }}$, wavenumbers $k$, and focal spot sizes $w_{0}$ is shown in Fig. 3. It can be seen that $w_{2} / w_{0}$ and $d$ approximately obey the relation 

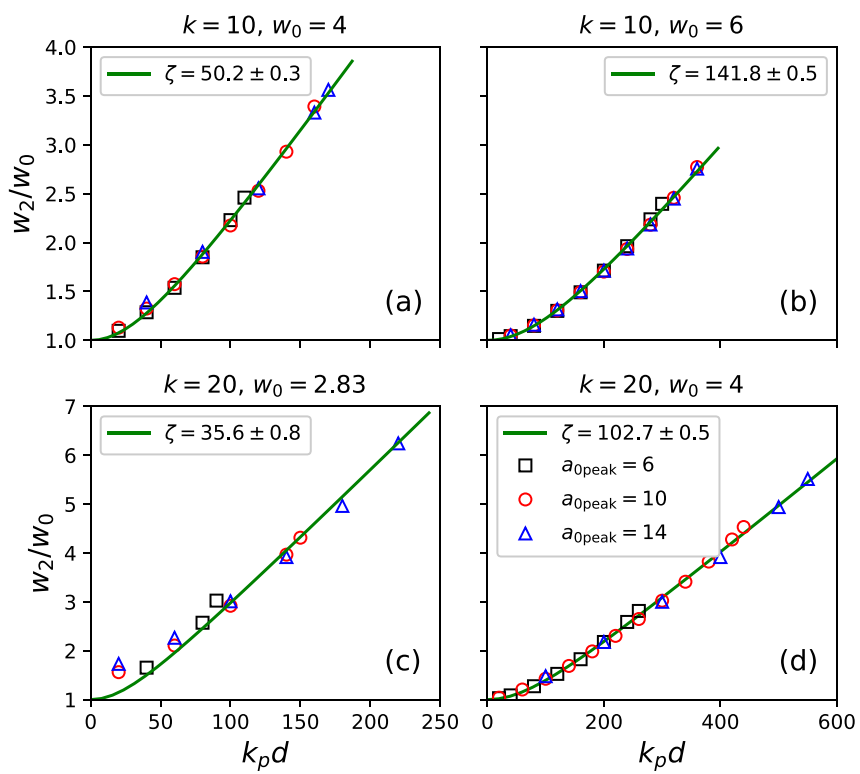

FIG. 3. The effective focal size-vacuum focal size ratio $w_{2} / w_{0}$ vs the distance from the vacuum focus to the plasma $d$ for $k=10$ (top) and $k=20$ (bottom). In each of the subplots, $k$ and $w_{0}$ are fixed, while $a_{0 \text { peak }}$ varies from 6 (black squares), 10 (red circles) to 14 (blue triangles). The green lines are the fits by Eq. (11), with the parameter $\zeta$ shown in the legends.

$$
\frac{w_{2}}{w_{0}}=\sqrt{1+\frac{d^{2}}{\zeta^{2}}},
$$

where $\zeta$ is a parameter that depends on $k$ and $w_{0}$, but only weakly on $a_{0 \text { peak }} \zeta$ can be regarded as a modified Rayleigh length to be discussed in Sec. IV. As is evident from Fig. 3(c), when $d$ is very small, the behavior of $w_{2} / w_{0}$ deviates from the general trend. This is caused by large cavitation (LC), occurring when $a>w^{2}$ as described in Appendix B.

A weak dependence of $\zeta$ on $a_{0 \text { peak }}$ appears over a large parameter range. Although it may be not intuitively understandable, such a weak dependence is a natural requirement for self-refocusing to occur. Due to the laser pulse having a limited duration, $a_{0}(\xi)$ changes along its temporal profile with the center having a maximum value of $a_{0 \text { peak }}$, whereas $a_{0}(\xi)$ is smaller at the front and rear of the pulse. Were $\zeta$ to have a strong dependence on $a_{0}$, self-refocusing of different temporal slices would be different, rendering a global uniform refocusing of the laser pulse impossible. In other words, only in the regime where such weak dependence is satisfied, can self-refocusing be observed.

The variation of $\zeta$ with $z_{R}$ and $k$ is plotted in Fig. 4(a). For a fixed value of $k$ a linear dependence of $\zeta$ on $z_{R}$ is observed. Thus the data in Fig. 4(a) can be fitted with $\zeta=\alpha z_{R}+\beta$, with the resulting fit parameters $\alpha$ and $\beta$ shown in Fig. 4(b). ${ }^{32}$ The dependence of $\alpha$ and $\beta$ on $k$ is again found from a linear fit as

$$
\begin{gathered}
\alpha=(-0.0027 \pm 0.0017) k+(0.950 \pm 0.028), \\
\beta=(-1.17 \pm 0.20) k+(-12.6 \pm 2.7) .
\end{gathered}
$$

The expressions for $\alpha$ and $\beta$ then allow for an empirical expression for $\zeta$ to be obtained:
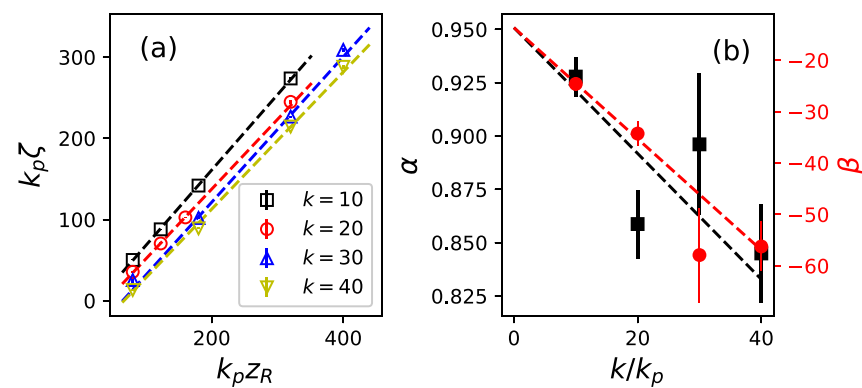

FIG. 4. (a) The modified Rayleigh length $\zeta$ as a function of vacuum Rayleigh length $z_{R}$ with $k=10$ (black squares), 20 (red circles), 30 (blue triangles), and 40 (yellow inverted triangles). (b) The linear fit parameters $\alpha$ (black squares) and $\beta$ (red dots) vs $k$, where the fit function is $\zeta=\alpha z_{R}+\beta$. The dashed lines are the linear fits, and the vertical lines are the error bars.

$$
\zeta \approx 0.95 z_{R}-1.2 k-13,
$$

where the $k$-dependence of $\alpha$ is neglected, since for $k \leq 40$ the first term on the RHS of Eq. (12) is less than $10 \%$ of the second term.

Note that if $z_{R}$ is not large enough so that $\zeta \leq 0$, a local maximum of $w$ can never be reached regardless of the value of $d$. Also when $\zeta$ is positive but small, $w_{2}$ is sensitive to variations of $d$, because $\delta w_{2}^{2}=w_{0}^{2} \zeta^{-2} \delta d^{2}$.

\section{B. Plasma eyepiece thickness vs prefocusing distance}

Some examples of the plasma eyepiece thickness $l$ as a function of the prefocusing distance $d$ are plotted in Fig. 5. It is evident that $l$ is almost proportional to $d$ for a fixed $k$ and $w_{0}$. Some exceptions are seen in Fig. 5(c) for small values of $d$, again due to large cavitation occurring when $a>w^{2}$ as described in Appendix B. The variation of plasma eyepiece thickness $l$ with $d$ is modeled with the relation
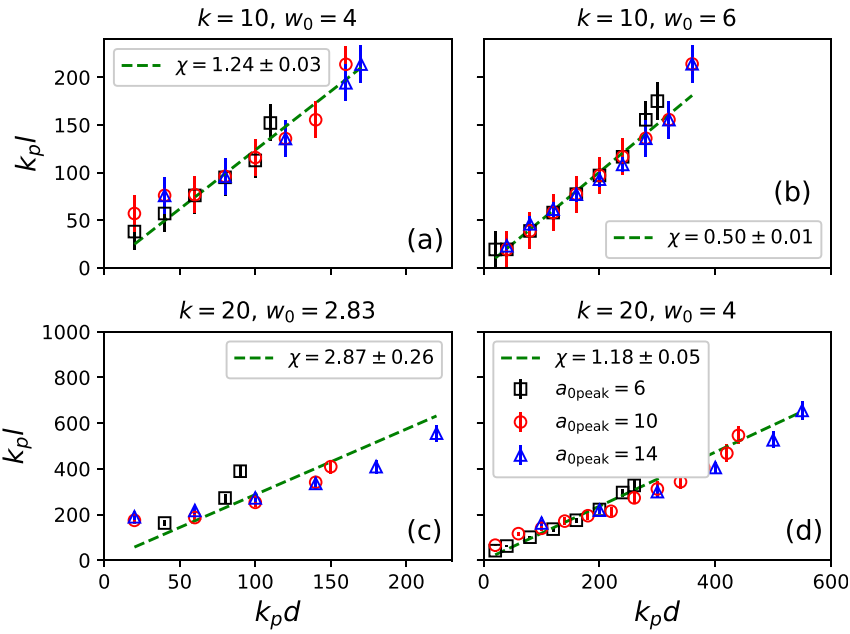

FIG. 5. Plasma eyepiece thickness $/$ as a function of the prefocusing distance $d$ for different values of $k, w_{0}$, and $a_{0 \text { peak }}=6$ (black squares), 10 (red circles), and 14 (blue triangles). The vertical lines are error bars due to the simulation dumping intervals. The green dashed lines are fits by Eq. (15), with the slope $\chi$ shown in the legends. 


$$
l=\chi d,
$$

where $\chi$ is a fit parameter depending on both $k$ and $w_{0}$.

The variation of $\chi$ with the vacuum spot size $w_{0}$ is then plotted in Fig. 6. As can be seen, all data points lie approximately on the same curve regardless of the value of $k$. An expression for $\chi$ can now be written as

$$
\chi=\mu w_{0}^{\nu}
$$

and performing a fit to the data presented in Fig. 6 results in $\mu=21.0 \pm 1.8$ and $\nu=-2.08 \pm 0.05$. Thus,

$$
\chi \approx 21.0 w_{0}^{-2.08}
$$

and the final empirical formula for the plasma eyepiece thickness $l$ is obtained as

$$
l \approx 21.0 \frac{d}{w_{0}^{2.08}}
$$

\section{Limitation of the prefocusing distance}

Although in all of our simulations the laser powers were chosen to be higher than the critical powers for relativistic self-focusing ${ }^{29}$ $\left[P / P_{c}=a_{0}^{2} w_{0}^{2} / 32>1\right.$ so that the RHS of Eq. (4) is negative], selfrefocusing does not always occur because the initial conditions in our case $d w /\left.d z\right|_{z_{1}}>0$ may lead to monotonic increasing $w$. An example of self-refocusing not occurring is shown in Fig. 7, where $k, w_{0}$ and $a_{0 \text { peak }}$ are fixed while $d$ changes from 420 to 450 . It can be seen that for $d=420$ and 440 the local maxima of $w$ exist while for $d=450$ the curve does not have a local maximum. Thus the threshold value of $d$ for self-refocusing to occur is approximately 445 , or $d_{\lim }=445 \pm 5$ in this case.

In Table II, some values of the threshold for refocusing obtained from simulation results $d_{\lim }$ are compared with the analytical results $d_{\mathrm{M}}$ from Eq. (9). One can see that in some of the cases, specifically for $a_{0 \text { peak }}=6, d_{\text {lim }}$ agrees with $d_{\mathrm{M}}$ reasonably well, while in the other cases $d_{\mathrm{M}}>d_{\text {lim. }}$. Finding the general expression for $d_{\text {lim }}$ is computationally expensive; thus, we take $d_{\mathrm{M}}$ to represent $d_{\text {lim }}$.

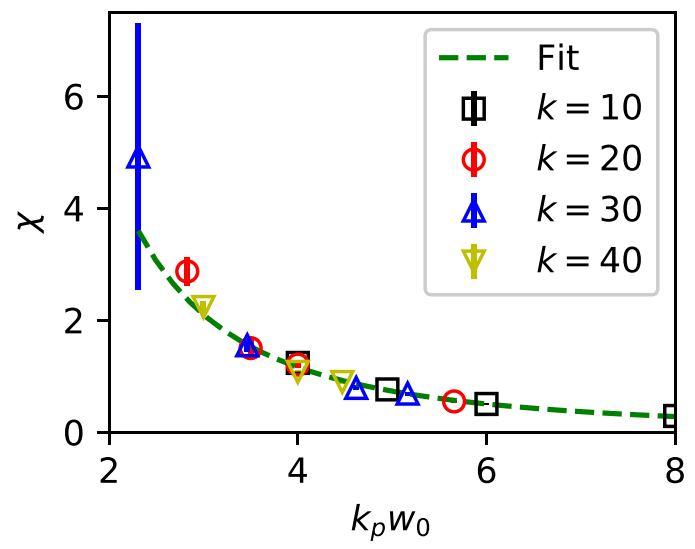

FIG. 6 . The ratio $\chi=I / d$ as a function of the vacuum focal size $w_{0}$ for $k=10$ (black squares), 20 (red circles), 30 (blue triangles), and 40 (yellow inverted triangles). The green dashed line is the fit of all the data by Eq. (16).

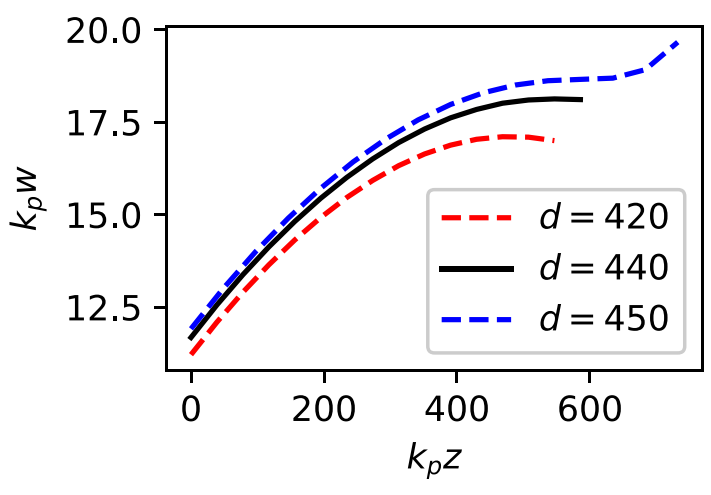

FIG. 7. Examples of laser spot size evolution, showing the threshold of $d$ for selfrefocusing to occur. The parameters are $k=20, w_{0}=4$, and $a_{\text {opeak }}=10$. The three curves are for $d=420$ (red dashed line), 440 (black solid line), and 450 (blue dashed line).

\section{Influence of pulse duration}

In the previous discussion, we took the averaged information of the laser temporal slices. The slice differences can be small if the laser beam has a long pulse duration. However, in short pulse cases, the slice differences may be important. Thus, in the following, we discuss pulse duration effects.

Figure 8 shows the influence of changing the initial pulse duration $\tau$ for two example cases. It is evident that with $\tau$ increasing from 2 to 19 , both $w_{2}$ and $l$ decrease. The influence of $\tau$ is stronger at smaller values of $\tau$. This is due to a shorter laser pulse creating a higher gradient in the plasma electron density, which then has a higher impact on the laser evolution. In a longer laser pulse case, only the front of the laser experiences a high electron density gradient, while the majority of the laser pulse is in a relatively longitudinally uniform density region. This is the physical reason behind the effect of changes of $\tau$ becoming less severe for longer values of the pulse length.

Although the refocusing effect changes with pulse duration $\tau$, the resulting variation of $w_{2}$ is less than $10 \%$ for all cases. The variation of $l$ is large, but less influential as explained in the following. Because $d w / d z$ approaches 0 while $w$ approaches $w_{2}$, the change of $w$ near the maximum is too small to ensure an exact measurement of $l$, resulting in a larger error in $l$. Meanwhile, because $l$ is usually much shorter than the limiting length scales in LWFAs as shown in Sec. V, knowing the exact value of the plasma eyepiece thickness $l$ is less important.

TABLE II. Comparisons of the threshold for refocusing obtained from simulations $d_{\text {lim }}$ and from the analytical model $d_{M}$.

\begin{tabular}{cccccccccc}
\hline \hline$k$ & $w_{0}$ & $a_{0 \text { peak }}$ & $d_{\lim }( \pm 5)$ & $d_{\mathrm{M}}$ & $k$ & $w_{0}$ & $a_{0 \text { peak }}$ & $d_{\lim }( \pm 5)$ & $d_{\mathrm{M}}$ \\
\hline 10 & 4 & 6 & 115 & 150 & 20 & 2.83 & 6 & 105 & 89 \\
& 10 & 165 & 271 & & & 10 & 155 & 183 \\
& 14 & 175 & 388 & & 14 & 225 & 268 \\
& 6 & 305 & 544 & 4 & 6 & 265 & 299 \\
& 10 & 365 & 937 & & 10 & 445 & 542 \\
& 14 & 365 & 1324 & & 14 & 595 & 776 \\
\hline
\end{tabular}




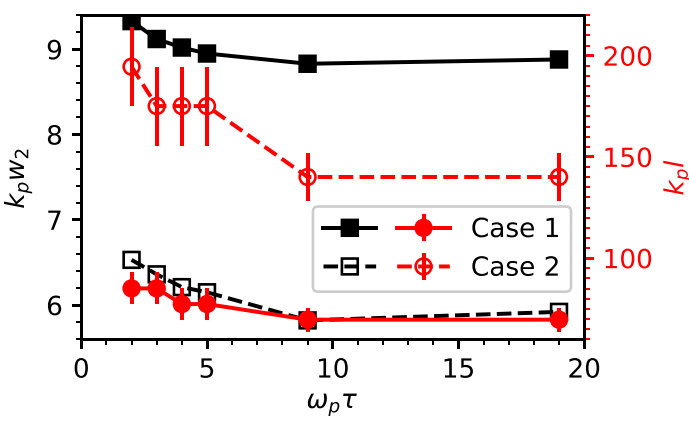

FIG. 8. The effective focal size $w_{2}$ and the plasma eyepiece thickness / vs the laser pulse duration $\tau$ for two cases. Case 1: $k=10, w_{0}=6, a_{0 \text { peak }}=14, d=160$ (solid lines and solid markers). Case 2: $k=20, w_{0}=3.5, a_{0 \text { peak }}=10, d=100$ (dashed lines and hollow markers). The black color is for $w_{2}$, the red color is for $I$, and the vertical lines on the red markers are the error bars due to the simulation dumping intervals.

In all the simulations of Secs. III A-III C, we have chosen $\tau=4$, where $w_{2}$ and $l$ approximately take the median values with respect to what is shown in Fig. 8. Thus, we consider our former conclusions reliable for a wide range of $\tau$, i.e., $\tau \geq 2$, which covers the resonant condition $\tau \sim \pi$.

\section{COMPARISON OF THE SIMULATION RESULTS, ANALYTICAL MODEL AND THE EMPIRICAL FORMULAS}

By comparing Eqs. (7) and (11), an analytical correspondence of $\zeta$ can be written as

$$
\zeta_{a}=z_{R} \sqrt{1-\left(1+\frac{d^{2}}{z_{R}^{2}}\right) \frac{32}{a_{0}^{2} w_{0}^{2}}} .
$$

This suggests that $\zeta_{a}$ mainly depends on but is smaller than the Rayleigh length $z_{R}$ which is similar to the empirical expression for $\zeta$ in Eq. (14). Also by comparing Eqs. (8) and (15), an analytical correspondence of $\chi$ can be found as

$$
\chi_{a}=\frac{1}{\frac{a_{0}^{2} w_{0}^{2}}{32}\left(1+\frac{d^{2}}{z_{R}^{2}}\right)^{-1}-1} .
$$

In the limit of the laser power being much higher than the critical power for self-focusing $a_{0}^{2} w_{0}^{2} / 32 \gg 1,{ }^{29}$ applying the weakly relativistic approximation $a_{0} \leqq 1$ (thus $w_{0} \gg 1$ ) and with the assumption of short distance from vacuum focus to plasma $d \ll z_{R}$, Eq. (20) reduces to $\chi_{a} \propto w_{0}^{-2}$, which is again similar to the empirical expression for $\chi$ in Eq. (17).

The variation of the refocused spot size $w_{2}$ and the plasma eyepiece thickness $l$ with prefocusing distance $d$ in both the empirical formulas and the analytical model (by setting $a_{0}=\bar{a}_{0}=a_{0 \text { peak }} / 2$ ) is compared to some simulations in Fig. 9. It is evident that in most cases the empirical formulas fit the simulations well, but the analytical model only partially agrees with the simulations for a smaller $a_{0 \text { peak }}$. The disagreement arises from the fact that for $a_{0 \text { peak }} \gg 1$, the plasma cannot be regarded as unperturbed; thus, the weekly relativistic assumption used in the analytical model is no longer applicable.
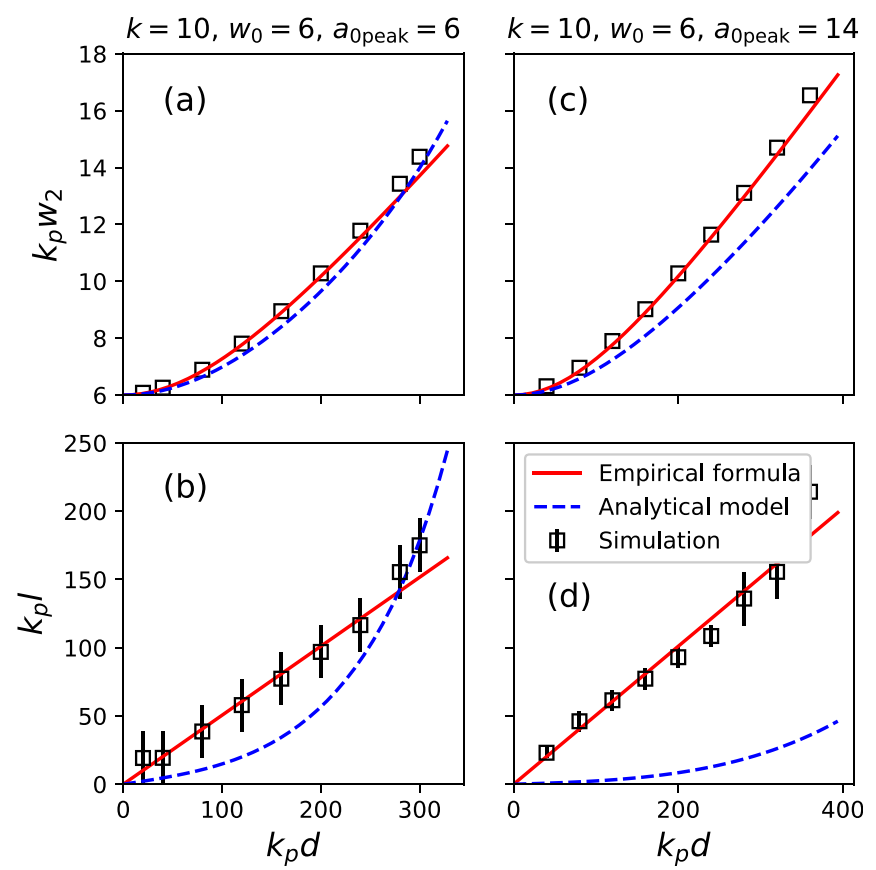

FIG. 9. Comparison of empirical formulas Eqs. (11), (14), and (18) (red solid lines), the analytical model Eqs. (7) and (8) (blue dashed lines), with the example simulation results (black squares). The parameters are $k=10, w_{0}=6$, and $a_{\text {opeak }}=6$ for (a) and (b), and 14 for (c) and (d). (a) and (c) compare the effective spot size $w_{2}$, while (b) and (d) compare the plasma eyepiece thickness $I$.

\section{FULL SCALE LWFA SIMULATIONS}

In order to show the physical scales of the plasma eyepiece explicitly, unnormalized units will be used in this section. Using Eqs. (11), (14), and (18), and applying the matching condition ${ }^{12}$ at $z_{2}$ (i.e., $k_{p} w_{2}=2 \sqrt{a_{2 \text { peak }}}$ where $a_{2 \text { peak }}$ is the peak normalized laser vector potential amplitude at $z_{2}$ ), a plasma eyepiece is designed for a $1 \mathrm{PW}$, $800 \mathrm{~nm}$ laser with $108 \mathrm{fs}$ pulse duration. A linear density ramp from $z=-20 k_{p}^{-1}=-216 \mu \mathrm{m}$ to $z_{1}=0$ is followed by a density plateau with density $n_{p}=2.43 \times 10^{17} \mathrm{~cm}^{-3}$ (thus $k_{p}^{-1}=10.8 \mu \mathrm{m}$ and $\omega_{p}^{-1}=35.9 \mathrm{fs}$ ). The laser frequency is thus $\omega / \omega_{p}=k / k_{p}=84.7$. The FWHM pulse duration is $\tau=3 \omega_{p}^{-1}$ in order to match the pumpdepletion length to the dephasing length, ${ }^{12}$ and the laser vacuum focus is set to $z_{0}=-80 k_{p}^{-1}=-864 \mu \mathrm{m}$ with $w_{0}=2 k_{p}^{-1}=21.6 \mu \mathrm{m}$, thus $a_{0 \text { peak }}=8$. The influence of the density transition region is neglected; thus, the prefocusing distance is $k_{p} d=80$. According to Eqs. (11), (14), and (18), this setup results in $w_{2}=4 k_{p}^{-1}=43.2 \mu \mathrm{m}$ and $a_{2 \text { peak }}=4$, with plasma eyepiece thickness $l=397 k_{p}^{-1}=4.29 \mathrm{~mm}$.

A simulation has been performed with the parameters discussed above, setting the simulation box size to $17 k_{p}^{-1} \times 40 k_{p}^{-1} \times 40 k_{p}^{-1}$ and number of cells to $4096 \times 256 \times 256$. The time step is $\Delta t=4.147$ $\times 10^{-3} \omega_{p}^{-1}$. Snapshots of a transverse slice through the laser pulse and laser spot size/amplitude evolution are shown in the top panels of Fig. 10. At around $z=292 k_{p}^{-1}$, the laser size $w$ is increased to $4 k_{p}^{-1}$ and the laser peak amplitude $a_{\text {peak }}$ is reduced to $4 .{ }^{33}$ At later times, the 

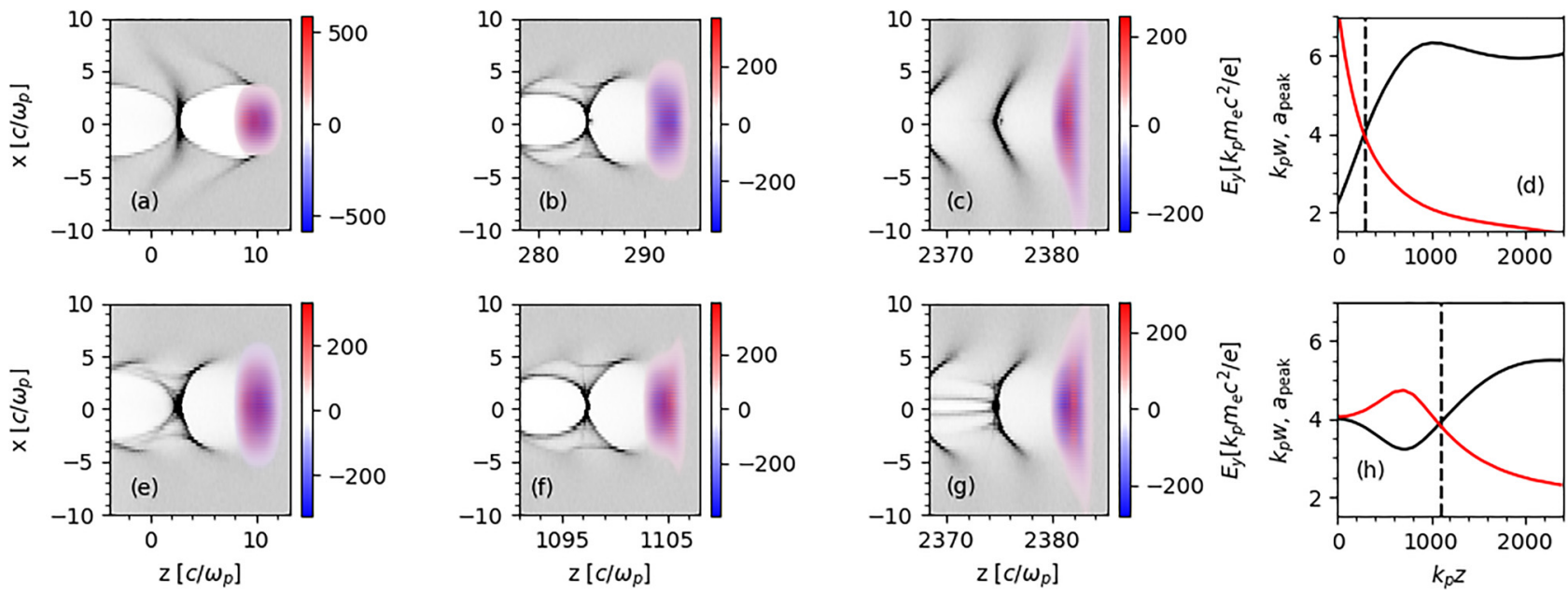

FIG. 10. Two 2-cm-long 3D PIC simulations showing the LWFAs with or without a plasma eyepiece. Top: with plasma eyepiece. The laser beam has $k_{p} w_{0}=2$ and is focused at $z=-80 k_{p}^{-1}$. Lu's matching condition is satisfied at around $z=292 k_{p}^{-1}$. Bottom: without plasma eyepiece. The laser beam has $k_{p} w_{0}=4$ and focused at $z=0$. Lu's matching condition is satisfied at $z=0$. The pseudocolor plots show the side views of the laser beams and the plasma densities, and the right-most plots show the evolution of the laser beam front-view size ( $w$, black lines) and the laser peak amplitude ( $a_{\text {peak }}$, read lines). The locations of the snapshots taken in (b) and ( $f$ ) are marked as dashed lines in (d) and (h). An animation showing the whole process is available in the supplementary material.

laser front becomes not well guided and at around $z=2000 k_{p}^{-1}$ the spot size $w$ stabilizes at about $5.9 k_{p}^{-1}$. The mismatch between the measured $\left(w_{2}, l\right)$ and the prediction from the empirical formulas has two reasons. One is the change of pulse duration: $\omega_{p} \tau=3$ is used here while the value $\omega_{p} \tau=4$ was used for obtaining the empirical formulas. The other is the error from the extrapolation of the results for $10 \leq k / k_{p} \leq 40$ to $k / k_{p}=84.7$. In a real experiment, one can avoid these errors by scanning $d$ around the value given by our empirical formula. Nevertheless, the blowout radius remains at about $4 k_{p}^{-1}$ for later times. Although a small bunch of electrons with a charge of $16 \mathrm{pC}$ is self-injected in the wake, the charge of this bunch is negligible compared to the loading capacity of this wake which is a few nanocoulombs according to Eq. (10) of Ref. 12 and Eq. (10) of Ref. 34. Moreover, according to Eqs. (3) and (4) of Ref. 12, both the pump depletion length $l_{\text {dep }} \approx\left(k / k_{p}\right)^{2} \omega_{p} \tau k_{p}^{-1}=2.15 \times 10^{4} k_{p}^{-1}=232 \mathrm{~mm}$ and the dephasing length $l_{d} \approx \frac{2}{3}\left(k / k_{p}\right)^{2} w_{2}=1.91 \times 10^{4} k_{p}^{-1}=206 \mathrm{~mm}$ are much larger than $l$; thus, the plasma eyepiece does not significantly shorten the acceleration length.

To highlight the performance of the plasma eyepiece, a simulation without the plasma eyepiece was performed with $a_{0 \text { peak }}=4$, $w_{0}=4 k_{p}^{-1}=43.2 \mu \mathrm{m}$ and $k_{p} d=0$. The results of this simulation are shown in the bottom panels in Fig. 10. The spot size $w$ and laser peak amplitude $a_{\text {peak }}$ evolution, shown in Figs. 10(d) and 10(h) for the simulation with and without the plasma eyepiece, respectively, have differences for the two cases. Nevertheless, the laser spot size approaches $k_{p} w=5.5$ in the case without the plasma eyepiece, which is similar to the value of $k_{p} w=5.9$ for the simulation with the plasma eyepiece. The spot size does not become stabilized at the matched value of 4 . This is due to the front of the laser being not well guided. Moreover, a bunch of electrons is also injected due to the evolution of the driver in this case, with the charge $15 \mathrm{pC}$ being similar to that in the former case.
The similarities in the results of the two cases clearly highlight the usefulness of the plasma eyepiece. For the 1 PW laser simulated, reaching a spot size of $w_{0}=43.2 \mu \mathrm{m}$ without a plasma eyepiece requires a focal length of about $20 \mathrm{~m}$. By prefocusing the same laser to $w_{0}=21.6 \mu \mathrm{m}$ with a focal length of $10 \mathrm{~m}$ and employing the plasma eyepiece, the similar effective spot size is achieved.

\section{DISCUSSIONS}

One may notice $\zeta$ in Eq. (14) has to be positive, which gives a limit

$$
\sqrt{\frac{n_{p}}{n_{c}}}=\frac{k_{p}}{k}>\frac{6.8+\sqrt{47+1.3 k z_{R}}}{k z_{R}},
$$

where $n_{c}=k^{2} / 4 \pi r_{e}$ is the critical density for the laser. Moreover, although $a_{0 \text { peak }}$ does not noticeably influence the parameters $\zeta$ and $\chi$, it influences the upper limit of $d$ for the refocusing to occur. To find this upper limit by simulations is extremely consuming in computational resources. Nevertheless, we found the analytical correspondence $d_{\mathrm{M}}$ in Eq. (9) gives a satisfactory estimation for this limit specifically when $a_{0 \text { peak }} \leq 6$ as shown in Table II. Thus, we use Eq. (9) together with Eq. (11) and take $a_{0}=a_{0 \text { peak }} / 2$ to obtain the laser spot size limit to which the plasma eyepiece can adjust,

$$
1 \leq \frac{w_{2}}{w_{0}}<\sqrt{1+\left(\frac{a_{0 \text { peak }}^{2} k_{p}^{2} w_{0}^{2}}{128}-1\right) \frac{z_{R}^{2}}{\zeta^{2}}} .
$$

For example, a $1 \mathrm{PW}$ laser beam, being prefocused to $w_{0}=20 \mu \mathrm{m}$ (thus $a_{0 \text { peak }}=8.6$ ), can be adjusted by a plasma eyepiece with the density $n_{p}$ down to $2 \times 10^{17} \mathrm{~cm}^{-3}$. And if we fix the plasma density to $n_{p}=5 \times 10^{17} \mathrm{~cm}^{-3}$, the spot size $w_{2}$ can be adjusted up to $67 \mu \mathrm{m}$ just by shifting the prefocusing position up to $d_{\mathrm{M}} \approx 2.8 \mathrm{~mm}$ in front of the plasma region. 
TABLE III. Plasma eyepiece parameters for 10 and 100 PW laser driven LWFAs. The dephasing length $L_{d}$, the optimal pulse duration $\tau_{\text {opt }}$ for matching the pump depletion length with the dephasing length, and the energy gain $\Delta W$ for LWFAs according to Lu et al. are also shown. ${ }^{12}$

\begin{tabular}{lcccc}
\hline \hline$P(\mathrm{PW})$ & 10 & 10 & 100 & 100 \\
\hline$a_{2 \text { peak }}$ & 4 & 4 & 4 & 4 \\
$n_{p}\left(\mathrm{~cm}^{-3}\right)$ & $2.4 \times 10^{16}$ & $2.4 \times 10^{16}$ & $2.4 \times 10^{15}$ & $2.4 \times 10^{15}$ \\
$w_{0}(\mu \mathrm{m})$ & 30 & 40 & 60 & 70 \\
$w_{2}(\mu \mathrm{m})$ & 136 & 136 & 431 & 431 \\
$d(\mathrm{~mm})$ & 35.6 & 17.7 & 694 & 563 \\
$l(\mathrm{~m})$ & 0.98 & 0.27 & 49 & 29 \\
$L_{d}(\mathrm{~m})$ & 6.52 & 6.52 & 206 & 206 \\
$\tau_{\text {opt }}(\mathrm{fs})$ & 303 & 303 & 958 & 958 \\
$\Delta W(\mathrm{GeV})$ & 97.7 & 97.7 & 977 & 977 \\
\hline \hline
\end{tabular}

Some sets of plasma eyepiece parameters for LWFAs driven by $800 \mathrm{~nm}$ wavelength lasers with powers of 10 and 100 PW, resulting from our empirical expressions and satisfying the matching condition $k_{p} w_{2}=2 \sqrt{a_{2 \text { peak }}}$, are shown in Table III. Full 3D simulations to verify these parameter sets are, however, extremely consuming in computational resources, thus requiring fast algorithms such as the quasi3 $\mathrm{D}$ algorithm. ${ }^{35-37}$

For some setups with short laser pulses, it may be difficult to satisfy $\omega_{p} \tau>2$ as discussed in Sec. IIID. One can still apply our design by staging the plasma region: a plasma eyepiece stage with a high plasma density so that $\omega_{p} \tau>2$ is satisfied, and an acceleration stage with a long dephasing length (low plasma density). The $w_{2}$ adjusted in the first stage does not have to be the matched spot size of the first stage; instead it should be the matched spot size of the second stage. The location of some specific injection mechanism such as ionization injections, ${ }^{38-47}$ density transition injections, ${ }^{48-50}$ and optical injections, ${ }^{51-53}$ should be placed after the plasma eyepiece stage for stable electron injections into the wake.

\section{CONCLUSION}

A telescope system with a plasma eyepiece based on the selffocusing effect was introduced to overcome the experimental limitations for focusing high power lasers to a variety of spot sizes. With a prefocusing optical system to focus the laser to a waist of $w_{0}$, adjustable effective focal spot sizes $w_{2}>w_{0}$ can be achieved by changing the preplasma focusing distance $d$ and the plasma density $n_{p}$. Empirical formulas for $w_{2}$ and the plasma eyepiece thickness $l$ were developed as Eqs. (11), (14), and (18). The focal length can be reduced by the factor of $w_{2} / w_{0}$ if we compare this system with conventional focusing elements that result in the same laser spot size. Further reductions of the focal length may be achievable by cascading plasma eyepieces.

The upper limit of the effective focal spot size is determined by the upper limit of the prefocusing distance $d_{\text {lim }}$. We found the analytical correspondence $d_{\mathrm{M}}$ expressed by Eq. (9) presents a satisfactory estimation for $d_{\text {lim }}$ specifically when $a_{0 \text { peak }}$ is not large $\left(a_{0 \text { peak }} \leq 6\right)$; thus, the spot size range that the plasma eyepiece can adjust is given by Eq. (22). A more universal expression for $d_{\text {lim }}$ requires further studies.

In previously proposed plasma lenses in the nonblowout regime, where ponderomotive force is negligible $a / w^{2} \leqq 0.1$, a transversely preformed plasma can perfectly resize the laser beam. ${ }^{22-25}$ For absolute blowout cases Eq. (B10), the ponderomotive effect dominates and the laser beam cannot be focused by the plasma. Our scheme operates in the transition regime, with both relativistic self-focusing and partial blowout $\left(0.1 \lesssim a / w^{2}<1\right)$. It is suitable for resizing the laser beams at the petawatt level to moderate intensities for wakefield accelerations.

\section{SUPPLEMENTARY MATERIAL}

See the supplementary material for the laser-plasma evolution animation for the two cases in Fig. 10.

\section{ACKNOWLEDGMENTS}

We thank the OSIRIS consortium (IST/UCLA) for access to the OSIRIS code and acknowledge the use of the high-performance cluster (Maxwell) at DESY. We also gratefully acknowledge the Gauss Center for Supercomputing e.V. (www.gauss-centre.eu) for funding this project by providing computing time through the John von Neumann Institute for Computing (NIC) on the GCS Supercomputer JUWELS at Jülich Supercomputing Center (JSC). This work was supported by the Helmholtz MT ARD scheme and the Helmholtz ZT-0009 project.

\section{APPENDIX A: A DERIVATION OF THE EQUATION OF LASER SPOT SIZE}

In this section, we give a derivation to Eqs. (3) and (4) using calculus of variations similar to Anderson and Bonnedal, ${ }^{27}$ but with a more explicit form of plasma response, i.e., the RHS of Eq. (1).

If we assume the relativistic factor of an electron is mainly contributed by the quiver motion driven by the laser, i.e., $\gamma=\sqrt{1+|\tilde{a}|^{2} / 2}$, and write down the Lagrangian

$$
\begin{aligned}
L & =\frac{i}{2}\left(\tilde{a} \partial_{z} \tilde{a}^{*}-\tilde{a}^{*} \partial_{z} \tilde{a}\right)-\frac{1}{2 k}\left|\nabla_{\perp} \tilde{a}\right|^{2} \\
& -\frac{2}{k}\left[\left(1+\frac{|\tilde{a}|^{2}}{2}\right)^{\frac{1}{2}}-\frac{|\tilde{a}|^{2}}{4}-1\right],
\end{aligned}
$$

where $\tilde{a}$ is complex and $\tilde{a}^{*}$ is its conjugate. Thus, Eq. (1), in the case of unperturbed plasma density $n=1$, is equivalent to the extremum problem

$$
0=\delta \iint 2 \pi r d r \times d z \times L\left(z, r ; \tilde{a}, \tilde{a}^{*}, \partial_{z} \tilde{a}, \partial_{z} \tilde{a}^{*}, \nabla_{\perp} \tilde{a}, \nabla_{\perp} \tilde{a}^{*}\right),
$$

where we have assumed a cylindrical symmetry, $r$ is the radial axis, and the integral is taken in the whole space.

Next, we assume the laser holds the form Eq. (2), where $a=a(z), u=u(z)$, and $w=w(z)$. One should notice that in this section $a=|a| \exp (-i \varphi)$ is complex because it also contains the Gouy phase $\varphi(z)$. And, for convenience, we define

$$
F(\varrho)=\exp \left(-\varrho^{2}\right)
$$

where $\varrho \equiv r / w$; thus, the partial derivatives of $\tilde{a}$ are 


$$
\begin{gathered}
\partial_{z} \tilde{a}=\left(\frac{a^{\prime}}{a}+i u^{\prime} r^{2}-\frac{r}{w} \frac{w^{\prime}}{w} \frac{F^{\prime}}{F}\right) \tilde{a}, \\
\partial_{r} \tilde{a}=\left(i 2 u r+\frac{1}{w} \frac{F^{\prime}}{F}\right) \tilde{a} .
\end{gathered}
$$

With Eqs. (A4) and (A5), we rewrite Eq. (A1) as

$$
\begin{aligned}
L= & {\left[\frac{i}{2}\left(a a^{* \prime}-a^{*} a^{\prime}\right)+\frac{|a|^{2}}{2 k}\right] F^{2}+\left[u^{\prime}-\frac{2 u^{2}}{k}\right]|a|^{2} r^{2} F^{2} } \\
& -\frac{|a|^{2} F^{\prime 2}}{2 k w^{2}}-\frac{2}{k}\left(\sqrt{1+\frac{|a|^{2} F^{2}}{2}}-1\right) .
\end{aligned}
$$

Define a reduced Lagrangian by taking transverse integration

$$
\begin{aligned}
\mathscr{L} \equiv & \int 2 \pi r d r \times L \\
= & \beta_{1}\left[\frac{i}{2}\left(a a^{* \prime}-a^{*} a^{\prime}\right) w^{2}+\frac{|a|^{2} w^{2}}{2 k}\right] \\
& +\beta_{2}\left[u^{\prime}-\frac{2 u^{2}}{k}\right]|a|^{2} w^{4}-\beta_{3} \frac{|a|^{2}}{2 k}-Q \frac{2 w^{2}}{k},
\end{aligned}
$$

where

$$
\begin{gathered}
\beta_{1}=\int 2 \pi \varrho d \varrho \times F^{2}, \\
\beta_{2}=\int 2 \pi \varrho d \varrho \times \varrho^{2} F^{2}, \\
\beta_{3}=\int 2 \pi \varrho d \varrho \times F^{2}, \\
Q=\int 2 \pi \varrho d \varrho \times\left(\sqrt{1+\frac{|a|^{2} F^{2}}{2}}-1\right) .
\end{gathered}
$$

Thus, Eq. (A2) is reduced to

$$
0=\delta \int d z \times \mathscr{L}\left(z ; a, a^{*}, u, w, a^{\prime}, a^{* \prime}, u^{\prime}, w^{\prime}\right) .
$$

With the Euler-Lagrange equation, we obtain

$$
\begin{gathered}
\frac{\partial \mathscr{L}}{\partial a}-\frac{d}{d z} \frac{\partial \mathscr{L}}{\partial a^{\prime}}=0: \\
0=-\frac{2 w^{2}}{k} \partial_{a} Q+\beta_{1}\left(i a^{* \prime} w^{2}+i a^{*} w w^{\prime}+\frac{a^{*} w^{2}}{2 k}\right) \\
+\beta_{2}\left(u^{\prime}-\frac{2 u^{2}}{k}\right) a^{*} w^{4}-\beta_{3} \frac{a^{*}}{2 k}, \\
\frac{\partial \mathscr{L}}{\partial u}-\frac{d}{d z} \frac{\partial \mathscr{L}}{\partial u^{\prime}}=0: \\
0=-\frac{4 u}{k}|a|^{2} w^{4}-\left(|a|^{2} w^{4}\right)^{\prime}, \\
\frac{\partial \mathscr{L}}{\partial w}-\frac{d}{d z} \frac{\partial \mathscr{L}}{\partial w^{\prime}}=0: \\
0=\beta_{1}\left[i\left(a a^{* \prime}-a^{*} a^{\prime}\right)+\frac{|a|^{2}}{k}\right] w+4 \beta_{2}\left(u^{\prime}-\frac{2 u^{2}}{k}\right)|a|^{2} w^{3}-4 Q \frac{w}{k} .
\end{gathered}
$$

By multiplying $a$ on both sides of Eq. (A13) and taking its imaginary part, we recover Eq. (3) (note $a \partial_{a} Q$ is real); by taking its real part and comparing with Eq. (A15), we have

$$
\beta_{2}\left(\frac{u^{\prime}}{2}-\frac{u^{2}}{k}\right)+\frac{\beta_{3}}{4 k w^{4}}=\frac{Q-a \partial_{a} Q}{k|a|^{2} w^{2}} .
$$

From Eqs. (3) and (A14), we obtain

$$
-\frac{2 u}{k}=\frac{w^{\prime}}{w},
$$

which also suggests $k / 2 u$ is the radius of the wavefront curvature. Thus,

$$
w^{\prime \prime}=\frac{\beta_{3}}{\beta_{2} k^{2} w^{3}}\left[1-\frac{4 w^{2}\left(Q-a \partial_{a} Q\right)}{\beta_{3}|a|^{2}}\right] .
$$

By inserting Eq. (A3) in Eqs. (A8)-(A11) one can obtain $\beta_{2}=\pi / 4$, $\beta_{3}=\pi$ and $Q-a \partial_{a} Q=\pi|a|^{4} / 128+\mathcal{O}\left(|a|^{6}\right)$. Finally, Eq. (4) is recovered.

One may notice the assumption that the laser holds the form Eq. (2) may not be correct, i.e., the laser may not perfectly maintain a Gaussian profile after propagating a while in plasma. In this case to define a laser radius $w$ is arbitrary. Thus Eq. (4) is just an approximate function for the laser spot size evolution in plasma.

\section{APPENDIX B: LARGE CAVITATION CONDITION}

When the ponderomotive force of the laser exceeds a threshold, the plasma electrons near the laser axis are completely evacuated because the static electric field cannot balance the ponderomotive force. Naturally one may conclude that in such condition, the laser at the cavitation area behaves as if it is in vacuum. Furthermore, if the cavitation radius is larger or equal to the laser size parameter $w$, almost the whole laser beam behaves as in vacuum.

We assume the laser beam is long enough so that only the transverse ponderomotive force is taken into consideration: ${ }^{26}$

$$
F_{p}=F_{p r}=-\frac{1}{4 \bar{\gamma}} \partial_{r} A^{2}=-\partial_{r} \bar{\gamma},
$$

where the force is normalized to $m_{e} c \omega_{p}, A=A(r)$ is the transverse normalized vector potential profile of a linear polarized laser beam, and $\bar{\gamma}=\sqrt{1+\frac{A^{2}}{2}}$ is the averaged Lorentz factor for the quivering electrons. If electron cavitation does not occur, i.e., electron density (normalized to plasma density $n_{p}$ ) $n_{e}>0$ everywhere, the transverse electrostatic force and the ponderomotive force balance each other $F_{p r}=E_{r}$. And from Gauss's law $\frac{1}{r} \partial_{r}\left(r E_{r}\right)=1-n_{e}$, one may find the relation between the electron density and the averaged Lorentz factor ${ }^{54}$

$$
1-n_{e}=-\frac{1}{r} \partial_{r}\left(r \partial_{r} \bar{\gamma}\right)=-\nabla_{\perp}^{2} \bar{\gamma}
$$

However, Eq. (B2) has nonphysical results when the laser ponderomotive force is stronger than the electrostatic force that an electronvacant ion column can provide. According to Gauss's law, an 
electron-vacant ion column has the transverse electrostatic field (normalized to $m_{e} c \omega_{p} / \mathrm{e}$ )

$$
E_{r}=\frac{1}{2} r \text { for } r \leq r_{c},
$$

where $r_{c}$ is the cavitation radius. If we assume the laser has a Gaussian profile

$$
A=a \exp \left(-\frac{r^{2}}{w^{2}}\right),
$$

one may expect cavitation to occur when

$$
\left.\partial_{r} F_{p}\right|_{r=0}>\left.\partial_{r} E_{r}\right|_{r=0}
$$

which leads to the cavitation condition

$$
\frac{a^{2}}{w^{2} \sqrt{1+\frac{a^{2}}{2}}}>\frac{1}{2} \text {. }
$$

Particularly, under the strongly relativistic condition $a^{2} \gg 1$, Eq. (B6) is approximated as

$$
\frac{a}{w^{2}}>\frac{1}{2 \sqrt{2}} .
$$

Moreover, when Eq. (B6) holds, the cavitation radius $r_{c}$ is obtained by applying $F_{p}=E_{r}$ at $r=r_{c}$ to Eqs. (B1), (B3), and (B4) as

$$
\frac{r_{c}^{2}}{w^{2}}=-\frac{1}{2} \ln \left[\frac{w^{2}}{16 a^{2}}\left(w^{2}+\sqrt{w^{4}+64}\right)\right] .
$$

We define the large cavitation condition to be $r_{c} \geq w$, so that the majority of the laser beam is in the electron-vacant ion column, which leads to

$$
\frac{w^{2}}{16 a^{2}}\left(w^{2}+\sqrt{w^{4}+64}\right) \leq \exp (-2) .
$$

This condition can be simplified if we assume $w^{2} \gg 1$; thus, we finally obtain the large cavitation (LC) condition

$$
\frac{a}{w^{2}} \geq 1
$$

where $w$ is normalized to $k_{p}^{-1}$.

The physical idea of the large cavitation condition is similar to the upper limit power for laser self-guiding. ${ }^{55}$ However, in Ref. 55, the averaged Lorentz factor $\bar{\gamma}$ was mainly contributed by the longitudinal motion of electrons, i.e., $\bar{\gamma} \propto A^{2}$. In our case the transverse motion of electrons dominates; thus, $\bar{\gamma} \propto A$.

One may notice that we use $a$ and $w$ without the subscription 0 because they can change during the propagation, and the cavitation and noncavitation states can switch. The matching condition by Lu et al. ${ }^{12}$ is $1 / 4=a_{0 \text { peak }} / w_{0}^{2}=2 a_{0} / w_{0}^{2}$; thus, $a_{0 \text { peak }}$ reaching the LC condition is 8 times the matching condition with the same $w_{0}$.

We show one example PIC simulation in Fig. 11. The initial parameters are $d=0, a_{0 \text { peak }}=50$ and $w_{0}=4$; thus, $a_{0}=\overline{a_{0}}$ $=a_{0 \text { peak }} / 2=25$ and Eq. (B10) is satisfied and large cavitation occurs at the vacuum-plasma interface. That is why for a short distance the laser size behaves similar to that in vacuum. One can
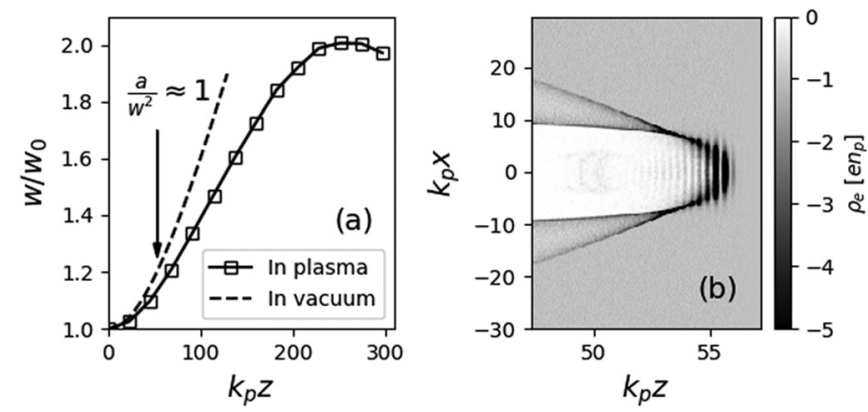

FIG. 11. An example simulation showing large cavitation with $k=10$, $w_{0}=4, a_{0 \text { peak }}=50$, and $d=0$ and the plasma region is at $z>0$ (thus, the laser is focused at the vacuum-plasma interface). (a) w vs $z$, for two cases: with plasma (square and solid line) and without plasma (dashed line). The arrow points out the approximate location where $a / w^{2}=1$. (b) The side slice view of the plasma electron density at where $a / w^{2} \approx 1$.

observe this similarity by comparing the solid line with the dashed line in Fig. 11(a). Then $a$ decreases and $w$ increases; at some distance $a / w^{2}=a_{\text {peak }} / 2 w^{2}=1$ appears. Although before $a / w^{2}=1$ occurs, the solid line does not exactly overlap with the dashed line, the location where $a / w^{2}=1$ is approximately the transition from "vacuum like" to "plasma like": before this point the $w$ vs $z$ curve behaves similar to that in vacuum, and after this point the curve presents selfrefocusing. Figure 11(b) shows the electron density slice at this transition point, in which a clear large electron-vacant column can be seen.

Furthermore, if we assume that the laser pulse evolves exactly the same as in vacuum for $a / w^{2}>1$, a further distance should be added to $d$ in the cases of $a_{1} / w_{1}^{2}>1$ :

$$
d_{\mathrm{eff}}=\max \left[d, z_{R} \sqrt{\left(\frac{a_{0}}{w_{0}^{2}}\right)^{\frac{2}{3}}-1}\right],
$$

where $d$ is still $z_{1}-z_{0}$, and $d_{\text {eff }}$ is the effective value replacing $d$ when using Eqs. (11) and (18). Correspondingly, $l$ also increases to its effective value

$$
l_{\mathrm{eff}}=l+d_{\mathrm{eff}}-d,
$$

where $l$ is obtained by Eq. (18) and $l_{\text {eff }}$ is total length of the plasma eyepiece. This explains why in Figs. 3(c) and 5(c) the $w_{2} / w_{0}$ and $l$ are slightly larger than the fit lines for some relatively small values of $d$.

\section{REFERENCES}

${ }^{7}$ See https://www.aip.org/fyi/2017/national-academies-study-aims-rally-usresearch-high-intensity-lasers for the broad interests and fast developments of petawatt laser facilities around the world.

${ }^{2}$ See https://www.nature.com/articles/nmat4533 for the ELI project.

${ }^{3}$ S. Gales, K. A. Tanaka, D. L. Balabanski, F. Negoita, D. Stutman, O. Tesileanu, C. A. Ur, D. Ursescu, I. Andrei, S. Ataman, M. O. Cernaianu, L. D’Alessi, I. Dancus, B. Diaconescu, N. Djourelov, D. Filipescu, P. Ghenuche, D. G. Ghita, C. Matei, K. Seto, M. Zeng, and N. V. Zamfir, "The extreme light infrastructure-nuclear physics (ELI-NP) facility: New horizons in physics with $10 \mathrm{PW}$ ultra-intense lasers and $20 \mathrm{MeV}$ brilliant gamma beams," Rep. Prog. Phys. 81, 094301 (2018).

${ }^{4}$ See https://www.clf.stfc.ac.uk/Pages/Vulcan-2020.aspx for the Vulcan 2020 project. 
$5^{5}$. H. Sung, H. W. Lee, J. Y. Yoo, J. W. Yoon, C. W. Lee, J. M. Yang, Y. J. Son, Y. H. Jang, S. K. Lee, and C. H. Nam, "4.2 pw, 20 fs ti:sapphire laser at $0.1 \mathrm{~Hz}$," Opt. Lett. 42, 2058-2061 (2017).

${ }^{6}$ L. Yu, Y. Xu, Y. Liu, Y. Li, S. Li, Z. Liu, W. Li, F. Wu, X. Yang, Y. Yang, C. Wang, X. Lu, Y. Leng, R. Li, and Z. Xu, "High-contrast front end based on cascaded xpwg and femtosecond opa for 10-pw-level ti:sapphire laser," Opt. Express 26, 2625-2633 (2018).

${ }^{7}$ See http://www.sciencemag.org/news/2018/01/physicists-are-planning-build-lasersso-powerful-they-could-rip-apart-empty-space for the 100-PW laser project SEL.

${ }^{8}$ L. Gallais, D.-B. Douti, M. Commandré, G. Batavičiùtė, E. Pupka, M. Sčiuka, L. Smalakys, V. Sirutkaitis, and A. Melninkaitis, "Wavelength dependence of femtosecond laser-induced damage threshold of optical materials," J. Appl. Phys. 117, 223103 (2015).

${ }^{9}$ T. Tajima and J. M. Dawson, "Laser electron accelerator," Phys. Rev. Lett. 43, 267-270 (1979).

${ }^{10}$ E. Esarey, C. B. Schroeder, and W. P. Leemans, "Physics of laser-driven plasma-based electron accelerators,” Rev. Mod. Phys. 81, 1229-1285 (2009).

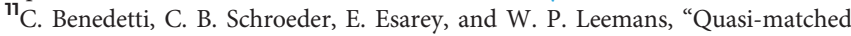
propagation of ultra-short, intense laser pulses in plasma channels," Phys. Plasmas 19, 053101 (2012)

${ }^{12}$ W. Lu, M. Tzoufras, C. Joshi, F. S. Tsung, W. B. Mori, J. Vieira, R. A. Fonseca, and L. O. Silva, "Generating multi-gev electron bunches using single stage laser wakefield acceleration in a 3d nonlinear regime," Phys. Rev. Spec. Top. Accel. Beams 10, 061301 (2007).

${ }^{13}$ I. Kostyukov, A. Pukhov, and S. Kiselev, "Phenomenological theory of laserplasma interaction in "bubble" regime," Phys. Plasmas 11, 5256-5264 (2004).

${ }^{14}$ K. Poder, J. M. Cole, J. C. Wood, N. C. Lopes, S. Alatabi, P. S. Foster, C. Kamperidis, O. Kononenko, C. A. Palmer, D. Rusby, A. Sahai, G. Sarri, D. R. Symes, J. R. Warwick, S. P. D. Mangles, and Z. Najmudin, "Measurements of self-guiding of ultrashort laser pulses over long distances," Plasma Phys. Controlled Fusion 60, 014022 (2018).

${ }^{15} \mathrm{M}$. Zeng and O. Tesileanu, "High-flux electron beams from laser wakefield accelerators driven by petawatt lasers," Plasma Sci. Technol. 19, 070502 (2017).

${ }^{16} \mathrm{~A}$. Macchi, M. Borghesi, and M. Passoni, "Ion acceleration by superintense laser-plasma interaction,” Rev. Mod. Phys. 85, 751-793 (2013).

${ }^{17}$ L. L. Ji, A. Pukhov, I. Y. Kostyukov, B. F. Shen, and K. Akli, "Radiation-reaction trapping of electrons in extreme laser fields," Phys. Rev. Lett. 112, 145003 (2014).

${ }^{18}$ I. C. E. Turcu, B. Shen, D. Neely, G. Sarri, K. A. Tanaka, P. McKenna, S. P. D. Mangles, T.-P. Yu, W. Luo, X.-L. Zhu et al., "Quantum electrodynamics experiments with colliding petawatt laser pulses," High Power Laser Sci. 7, e10 (2019).

${ }^{19}$ W. Luo, W.-Y. Liu, T. Yuan, M. Chen, J.-Y. Yu, F.-Y. Li, D. Sorbo, C. Ridgers, and Z.-M. Sheng, "Qed cascade saturation in extreme high fields," Sci. Rep. 8, 8400 (2018).

${ }^{20}$ W. Leemans, A. Gonsalves, H.-S. Mao, K. Nakamura, C. Benedetti, C. Schroeder, C. Tóth, J. Daniels, D. Mittelberger, S. Bulanov et al., "Multi-gev electron beams from capillary-discharge-guided subpetawatt laser pulses in the self-trapping regime," Phys. Rev. Lett. 113, 245002 (2014).

${ }^{21} \mathrm{C}$. E. Max, J. Arons, and A. B. Langdon, "Self-modulation and self-focusing of electromagnetic waves in plasmas," Phys. Rev. Lett. 33, 209-212 (1974).

${ }^{22}$ R. Hubbard, B. Hafizi, A. Ting, D. Kaganovich, P. Sprangle, and A. Zigler, "High intensity focusing of laser pulses using a short plasma channel lens," Phys. Plasmas 9, 1431-1442 (2002).

${ }^{23}$ Y. Katzir, S. Eisenmann, Y. Ferber, A. Zigler, and R. Hubbard, "A plasma microlens for ultrashort high power lasers," Appl. Phys. Lett. 95, 031101 (2009).

${ }^{24}$ J. Palastro, D. Gordon, B. Hafizi, L. Johnson, J. Peñano, R. Hubbard, M. Helle, and D. Kaganovich, "Plasma lenses for multi-petawatt laser pulses," Phys. Plasmas 22, 123101 (2015).

${ }^{25}$ D. F. Gordon, A. B. Stamm, B. Hafizi, L. A. Johnson, D. Kaganovich, R. F. Hubbard, A. S. Richardson, and D. Zhigunov, "Ideal form of optical plasma lenses," Phys. Plasmas 25, 063101 (2018).

${ }^{26}$ P. Mora, J. Thomas, and M. Antonsen, "Kinetic modeling of intense, short laser pulses propagating in tenuous plasmas," Phys. Plasmas 4, 217-229 (1997).

${ }^{27} \mathrm{D}$. Anderson and M. Bonnedal, "Variational approach to nonlinear selffocusing of gaussian laser beams," Phys. Fluids 22, 105-109 (1979).
${ }^{28}$ Z.-M. Sheng, Y. Cui, N. Cheng, and Y. Wei, "Photorefractive self-trapping and deflection of optical beams," J. Opt. Soc. Am. B 13, 584-589 (1996).

${ }^{29}$ P. Sprangle, C.-M. Tang, and E. Esarey, "Relativistic self-focusing of shortpulse radiation beams in plasmas," IEEE Trans. Plasma Sci. 15, 145-153 (1987).

${ }^{30} \mathrm{~K}$.-C. Tzeng and W. B. Mori, "Suppression of electron ponderomotive blowout and relativistic self-focusing by the occurrence of raman scattering and plasma heating," Phys. Rev. Lett. 81, 104-107 (1998).

${ }^{31}$ R. A. Fonseca, L. O. Silva, F. S. Tsung, V. K. Decyk, W. Lu, C. Ren, W. B. Mori, S. Deng, S. Lee, T. Katsouleas, and J. C. Adam, "Osiris: A three-dimensional, fully relativistic particle in cell code for modeling plasma based accelerators," in Proceedings of the International Conference on Computational Science (ICCS), edited by P. M. A. Sloot, A. G. Hoekstra, C. J. K. Tan, and J. J. Dongarra (Springer, Berlin, Heidelberg, 2002), pp. 342-351.

${ }^{32}$ In this paper, all the errors from the fits are the standard deviations if not explicitly specified.

${ }^{33} \mathrm{We}$ assume the laser wavelength does not change during this short-distance propagation so that $a_{\text {peak }}$ is proportional to the amplitude of the electric field. Thus $a_{\text {peak }}$ is obtained by taking the amplitude parameter of the Gaussian fit and normalizing to its value at the simulation start point.

${ }^{34}$ M. Tzoufras, W. Lu, F. S. Tsung, C. Huang, W. B. Mori, T. Katsouleas, J. Vieira, R. A. Fonseca, and L. O. Silva, "Beam loading in the nonlinear regime of plasma-based acceleration," Phys. Rev. Lett. 101, 145002 (2008).

${ }^{35}$ A. Lifschitz, X. Davoine, E. Lefebvre, J. Faure, C. Rechatin, and V. Malka, "Particle-in-cell modelling of laser-plasma interaction using fourier decomposition," J. Comput. Phys. 228, 1803-1814 (2009).

${ }^{36}$ A. Davidson, A. Tableman, W. An, F. S. Tsung, W. Lu, J. Vieira, R. A. Fonseca, L. O. Silva, and W. B. Mori, "Implementation of a hybrid particle code with a pic description in $\mathrm{r}-\mathrm{z}$ and a gridless description in $\phi$ into osiris," J. Comput. Phys. 281, 1063-1077 (2015).

${ }^{37}$ R. Lehe, M. Kirchen, I. A. Andriyash, B. B. Godfrey, and J.-L. Vay, "A spectral, quasi-cylindrical and dispersion-free particle-in-cell algorithm," Comput. Phys. Commun. 203, 66-82 (2016).

${ }^{38}$ M. Chen, Z.-M. Sheng, Y.-Y. Ma, and J. Zhang, "Electron injection and trapping in a laser wakefield by field ionization to high-charge states of gases," J. Appl. Phys. 99, 056109 (2006).

${ }^{39}$ E. Oz, S. Deng, T. Katsouleas, P. Muggli, C. D. Barnes, I. Blumenfeld, F. J. Decker, P. Emma, M. J. Hogan, R. Ischebeck, R. H. Iverson, N. Kirby, P. Krejcik, C. O'Connell, R. H. Siemann, D. Walz, D. Auerbach, C. E. Clayton, C Huang, D. K. Johnson, C. Joshi, W. Lu, K. A. Marsh, W. B. Mori, and M. Zhou, "Ionization-induced electron trapping in ultrarelativistic plasma wakes," Phys. Rev. Lett. 98, 084801 (2007).

${ }^{40}$ A. Pak, K. A. Marsh, S. F. Martins, W. Lu, W. B. Mori, and C. Joshi, "Injection and trapping of tunnel-ionized electrons into laser-produced wakes," Phys. Rev. Lett. 104, 025003 (2010).

${ }^{41}$ C. McGuffey, A. G. R. Thomas, W. Schumaker, T. Matsuoka, V. Chvykov, F. J. Dollar, G. Kalintchenko, V. Yanovsky, A. Maksimchuk, K. Krushelnick, V. Y. Bychenkov, I. V. Glazyrin, and A. V. Karpeev, "Ionization induced trapping in a laser wakefield accelerator," Phys. Rev. Lett. 104, 025004 (2010).

${ }^{42}$ J. S. Liu, C. Q. Xia, W. T. Wang, H. Y. Lu, C. Wang, A. H. Deng, W. T. Li, H. Zhang, X. Y. Liang, Y. X. Leng, X. M. Lu, C. Wang, J. Z. Wang, K. Nakajima, R. $\mathrm{X}$. Li, and Z. Z. Xu, "All-optical cascaded laser wakefield accelerator using ionization-induced injection," Phys. Rev. Lett. 107, 035001 (2011).

${ }^{43}$ B. B. Pollock, C. E. Clayton, J. E. Ralph, F. Albert, A. Davidson, L. Divol, C. Filip, S. H. Glenzer, K. Herpoldt, W. Lu, K. A. Marsh, J. Meinecke, W. B. Mori, A. Pak, T. C. Rensink, J. S. Ross, J. Shaw, G. R. Tynan, C. Joshi, and D. H. Froula, "Demonstration of a narrow energy spread, $\sim 0.5 \mathrm{GeV}$ electron beam from a two-stage laser wakefield accelerator," Phys. Rev. Lett. 107, 045001 (2011).

${ }^{44}$ M. Chen, E. Esarey, C. B. Schroeder, C. G. R. Geddes, and W. P. Leemans, "Theory of ionization-induced trapping in laser-plasma accelerators," Phys. Plasmas 19, 033101 (2012).

${ }^{45}$ M. Zeng, M. Chen, Z.-M. Sheng, W. B. Mori, and J. Zhang, "Self-truncated ionization injection and consequent monoenergetic electron bunches in laser wakefield acceleration," Phys. Plasmas 21, 030701 (2014).

${ }^{46}$ M. Zeng, M. Chen, L. L. Yu, W. B. Mori, Z. M. Sheng, B. Hidding, D. A. Jaroszynski, and J. Zhang, "Multichromatic narrow-energy-spread electron 
bunches from laser-wakefield acceleration with dual-color lasers," Phys. Rev. Lett. 114, 084801 (2015)

${ }^{47}$ M. Zeng, J. Luo, M. Chen, W. B. Mori, Z.-M. Sheng, and B. Hidding, "High quality electron beam acceleration by ionization injection in laser wakefields with mid-infrared dual-color lasers,” Phys. Plasmas 23, 063113 (2016).

${ }^{48} \mathrm{H}$. Suk, N. Barov, J. B. Rosenzweig, and E. Esarey, "Plasma electron trapping and acceleration in a plasma wake field using a density transition," Phys. Rev. Lett. 86, 1011-1014 (2001)

${ }^{49}$ C. G. R. Geddes, K. Nakamura, G. R. Plateau, C. Toth, E. Cormier-Michel, E. Esarey, C. B. Schroeder, J. R. Cary, and W. P. Leemans, "Plasma-density-gradient injection of low absolute-momentum-spread electron bunches," Phys. Rev. Lett. 100, 215004 (2008)

${ }^{50}$ A. Martinez de la Ossa, Z. Hu, M. J. V. Streeter, T. J. Mehrling, O. Kononenko, B. Sheeran, and J. Osterhoff, "Optimizing density down-ramp injection for beam-driven plasma wakefield accelerators," Phys. Rev. Accel. Beams 20, 091301 (2017).
${ }^{51}$ D. Umstadter, J. K. Kim, and E. Dodd, "Laser injection of ultrashort electron pulses into wakefield plasma waves," Phys. Rev. Lett. 76, 2073-2076 (1996).

${ }^{52}$ C. Rechatin, J. Faure, A. Ben-Ismail, J. Lim, R. Fitour, A. Specka, H. Videau, A. Tafzi, F. Burgy, and V. Malka, "Controlling the phase-space volume of injected electrons in a laser-plasma accelerator," Phys. Rev. Lett. 102, 164801 (2009).

${ }^{53}$ R. Lehe, A. F. Lifschitz, X. Davoine, C. Thaury, and V. Malka, "Optical transverse injection in laser-plasma acceleration," Phys. Rev. Lett. 111, 085005 (2013).

${ }^{54}$ G. Sun, E. Ott, Y. C. Lee, and P. Guzdar, "Self-focusing of short intense pulses in plasmas," Phys. Fluids 30, 526-532 (1987).

${ }^{55}$ W.-M. Wang, Z.-M. Sheng, M. Zeng, Y. Liu, Z.-D. Hu, S. Kawata, C.-Y. Zheng, W. B. Mori, L.-M. Chen, Y.-T. Li, and J. Zhang, "Upper limit power for selfguided propagation of intense lasers in plasma," Appl. Phys. Lett. 101, 184104 (2012). 